\title{
Role of Coherent Low-Frequency Motion in Excited-State \\ Proton Transfer of Green Fluorescent Protein Studied by \\ Time-Resolved Impulsive Stimulated Raman Spectroscopy
}

\author{
Tomotsumi Fujisawa ${ }^{1}$, Hikaru Kuramochi ${ }^{1}$, Haruko Hosoi ${ }^{2}$, Satoshi Takeuchi ${ }^{1,3}$, Tahei \\ Tahara, ${ }^{1,3 *}$ \\ ${ }^{1}$ Molecular Spectroscopy Laboratory, RIKEN, 2-1 Hirosawa, Wako 351-0198, Japan \\ ${ }^{2}$ Department of Biomolecular Science, Faculty of Sciences, Toho University, 2-2-1 Miyama, Funabashi 274-8510, Japan \\ ${ }^{3}$ Ultrafast Spectroscopy Research Team, RIKEN Center for Advanced Photonics (RAP), RIKEN, 2-1 Hirosawa, Wako \\ 351-0198, Japan
}

\section{Supporting Information}

1. Materials and method

2. Data analysis

3. Comparison of the vibrational spectra between the $A^{*}$ form and the ground state of GFP

4. Contribution of the vibrational coherence of the ground-state I form

5. Comparison between TR-ISRS and time-resolved IR spectra of A* and I* forms

6. Temporal change of a transient band of the I* form

7. Origin of the hot I* form

8. Comparison of the deviation of the early-time I* formation from the first order kinetics and the low-frequency spectrum of $A^{*}$

9. Formulation of the amplitude modulation of a high frequency Raman band through the anharmonic coupling with a low-frequency mode 


\section{Materials and method}

\section{Materials}

Aequorea victoria GFP was produced in E. coli and purified as described previously. ${ }^{1,2}$ A typical concentration of the sample solutions was $\sim 400 \mu \mathrm{M}$ in Tris buffer $(10 \mathrm{mM}$ Tris, $100 \mathrm{mM} \mathrm{NaCl}, 50$ $\mathrm{mM} \mathrm{Na}_{2} \mathrm{HPO}_{4}, \mathrm{pH} \mathrm{8.0)}$, which corresponds to the optical density of $\sim 0.8$ at $390 \mathrm{~nm}$ for 1-mm optical path length.

\section{Time-resolved impulsive stimulated Raman spectroscopy}

Details of the TR-ISRS setup is described elsewhere. ${ }^{3,4,5}$ Briefly, the output of the Ti:sapphire regenerative amplifier $(780 \mathrm{~nm}, 90 \mathrm{fs}, 1 \mathrm{~mJ}, 1 \mathrm{kHz})$ was split into two. The first portion was frequency doubled in a type-I BBO ( $\beta$-barium borate) crystal, generating the actinic pump pulse $\left(\mathrm{P}_{1}\right.$, $390 \mathrm{~nm}, 110 \mathrm{fs}$ ). The second portion of the amplifier output pumped a home-built noncollinear optical parametric amplifier (NOPA). The output of the NOPA (520-700 nm) was temporally compressed down to 6.5 fs by using the 4-f dispersion compensator equipped with a deformable mirror at the Fourier plane. The compressed pulse was divided into two; one was used as the impulsive Raman excitation $\left(\mathrm{P}_{2}\right)$, and the other was used as the probe pulse $\left(\mathrm{P}_{3}\right)$ to monitor the transient absorbance change. The spectra of the $\mathrm{P}_{1}, \mathrm{P}_{2}$ and $\mathrm{P}_{3}$ pulses are shown in Figure $\mathrm{S} 1$. All pulses were horizontally polarized. The $\mathrm{P}_{1}, \mathrm{P}_{2}$ and $\mathrm{P}_{3}$ pulses were focused into a $300-\mu \mathrm{m}$-thick flow cell of the sample solution. At the sample position, the energies of the $\mathrm{P}_{1}, \mathrm{P}_{2}$, and $\mathrm{P}_{3}$ pulses were 100,70 , and $5 \mathrm{~nJ}$.

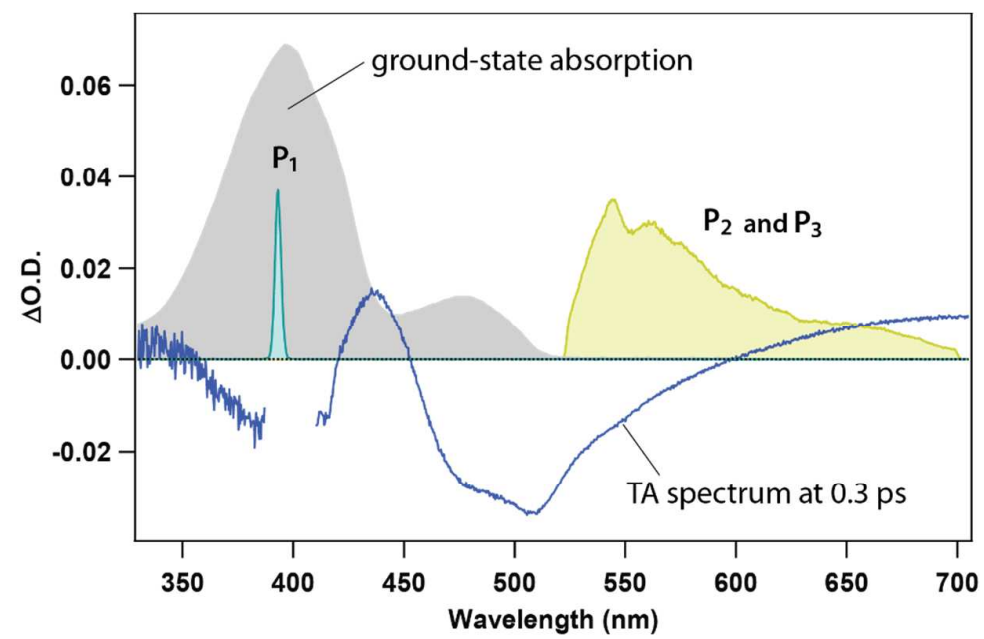

Figure S1. Spectra of the $P_{1}, P_{2}$, and $P_{3}$ pulses used in the TR-ISRS measurement of GFP. The absorption spectrum of GFP (gray) and the time-resolved absorption spectrum (TA spectrum, blue) at 0.3 ps are shown for comparison. 


\section{Data analysis}
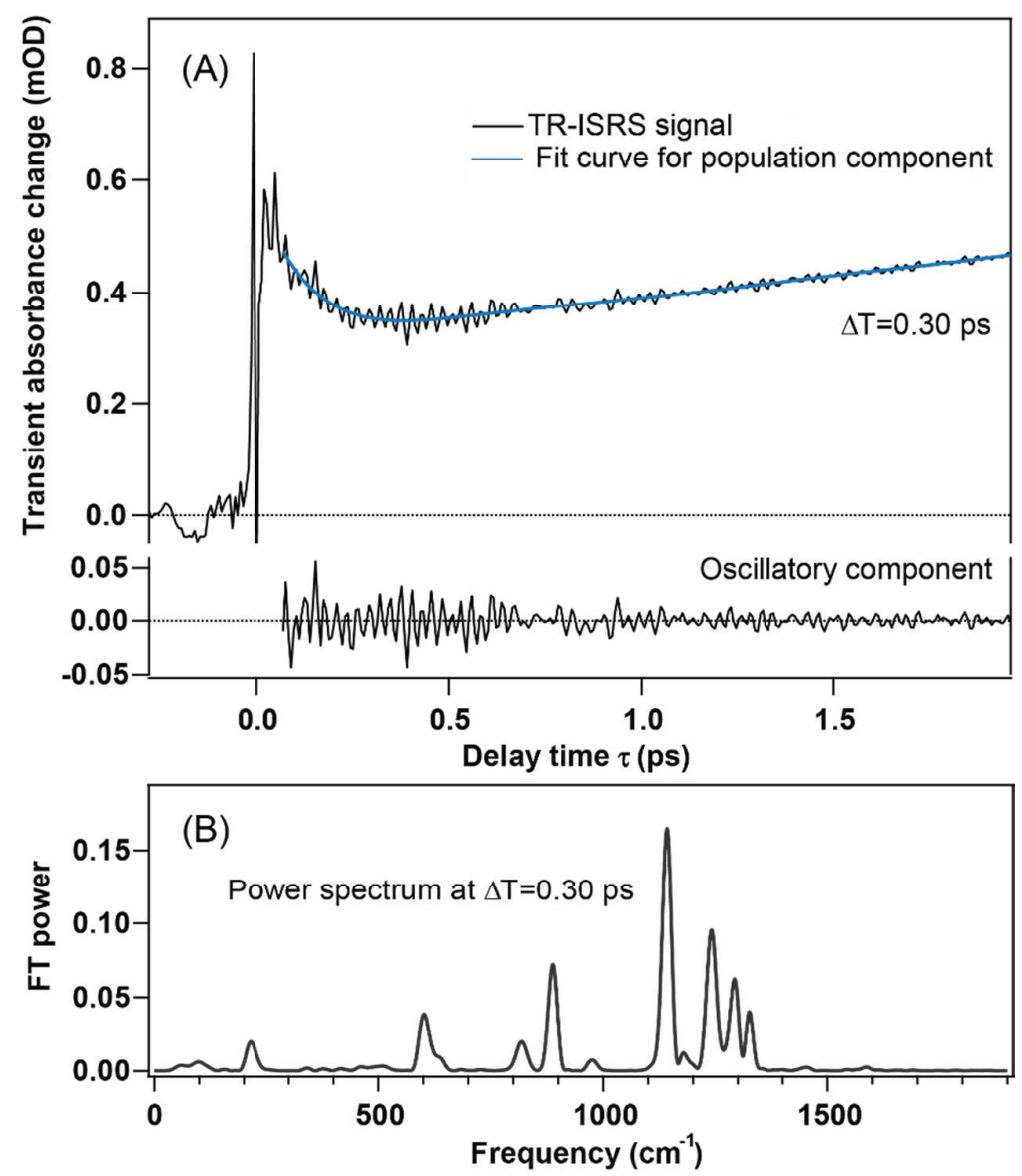

Figure S2. (A) Upper: TR-ISRS signal at $\Delta T=0.3 \mathrm{ps}$ and the fit curve for the slowly-varying population component (blue). Lower: oscillatory component obtained by subtracting the population component. (B) Power spectrum of the oscillatory component obtained by Fourier transform analysis.

To obtain the time-resolved vibrational spectrum from the raw TR-ISRS signal, the slowly-varying population component is first fitted with the 6th-order polynomial function (Figure S2A, upper). Then, this population component is subtracted from the raw data to obtain the oscillatory component (Figure S2A, lower). A frequency-domain vibrational spectrum is obtained by Fourier transformation of this time-domain signal after windowing and zero-padding. The peak positions, intensities, and bandwidths of the prominent bands are determined by Gaussian fitting. 


\section{Comparison of the vibrational spectra between the $A^{*}$ form and the ground state of GFP}

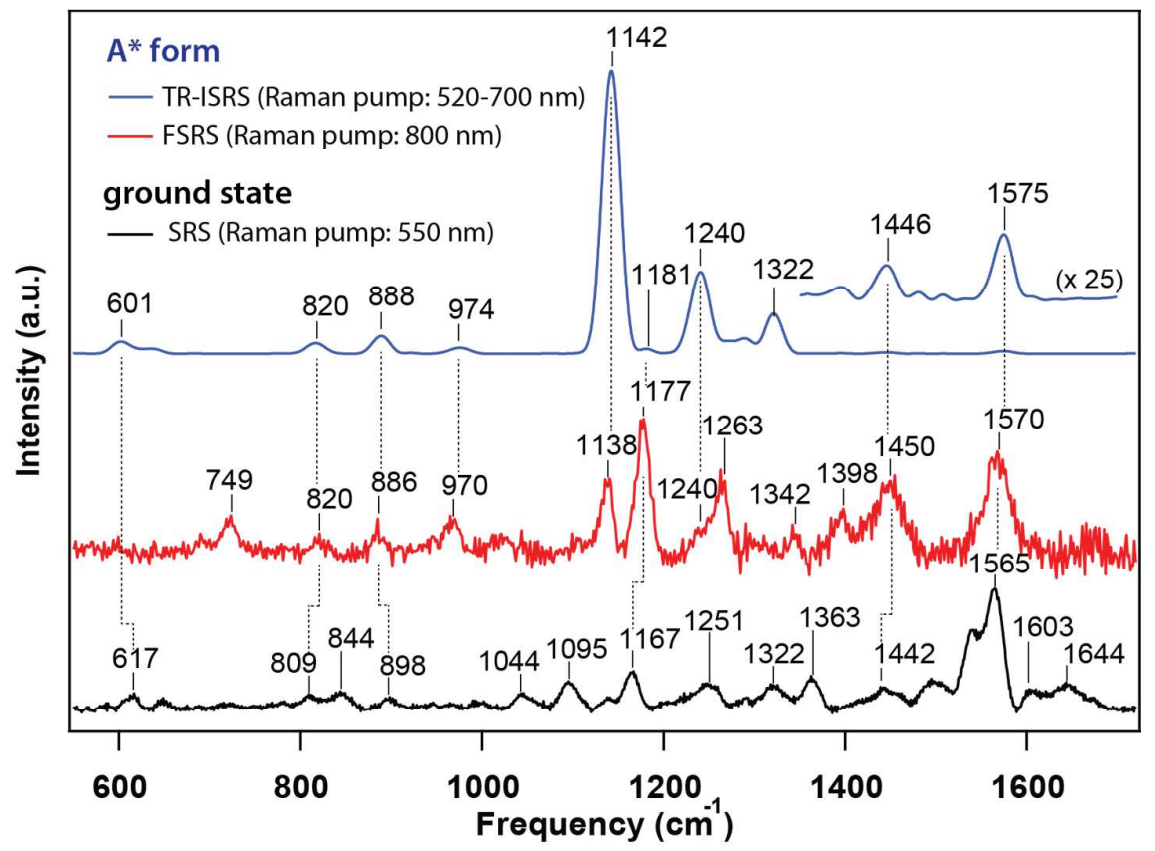

Figure S3. Blue: vibrational spectrum of the $A^{*}$ form obtained by TR-ISRS (Raman excitation: 520-700 $\mathrm{nm}$ ) at $\Delta \mathrm{T}=0.1 \mathrm{ps}$ after photoexcitation. Red: vibrational spectrum of the $A^{*}$ form obtained by FSRS (Raman excitation: $800 \mathrm{~nm}$ ) at 0.1 ps after photoexcitation. Black: steady-state stimulated Raman spectrum of GFP (Raman excitation: $550 \mathrm{~nm}$ ).

In Figure S3, the vibrational spectrum of the A* form obtained by TR-ISRS (blue) is compared with the FSRS spectrum of the $A^{*}$ form (red) as well as the stimulated Raman spectrum of the ground state (black). The FSRS spectrum of the A* form was obtained in the same way as ref. 6 using Raman pump at $800 \mathrm{~nm}$ and was measured at $0.1 \mathrm{ps}$ after photoexcitation. The vibrational spectrum of the ground state was obtained by the steady-state measurement using Raman pump at $550 \mathrm{~nm}$. Clearly, the vibrational frequencies of the $A^{*}$ form observed in TR-ISRS (blue) are different from the frequencies of the ground state (black) (except for the $\sim 1322 \mathrm{~cm}^{-1}$ mode) but are the same as those in the FSRS spectrum of the $A^{*}$ form within the experimental error. Actually, the vibrational bands at $820,888,974,1142,1240,1446$, and 1575 observed in TR-ISRS coincides with those in the FSRS spectrum of the $A^{*}$ form within $\pm 5 \mathrm{~cm}^{-1}$. This ensures that TR-ISRS selectively detects the vibrational modes of the $\mathrm{A}^{*}$ form. The difference between TR-ISRS and FSRS is seen in the relative intensities of vibrational bands of the $A^{*}$ form, which arises from their different resonance conditions; FSRS (Raman pump: $800 \mathrm{~nm}$ ) is resonant to the $\mathrm{S}_{\mathrm{n}} \leftarrow \mathrm{S}_{1}$ absorption while TR-ISRS (Raman pump: 520-700 nm) is resonant with the stimulated emission of the $A^{*}$ form (Figure S1). 


\section{Contribution of the vibrational coherence of the ground-state I form}

In the late $\Delta \mathrm{T}$ delay time region, the $\mathrm{P}_{2}$ pulse could create the vibrational coherence in the ground state of the I form through the stimulated emission transition, and it may contribute to the TR-ISRS signal. However, in the pump-dump-probe study by Kennis et. al., ${ }^{7}$ the ground state of the I form created by the dump pulse was found to show no absorption band over the spectral region of the $\mathrm{P}_{3}$ pulse $(520-700 \mathrm{~nm})$. Therefore, the $\mathrm{P}_{3}$ pulse in our TR-ISRS experiment cannot efficiently probe the ground-state vibrational coherence of the I form. The vibrationally hot ground-state I form, which can exist immediately after the dumping with the $\mathrm{P}_{2}$ pulse, may show absorption in the spectral range of the $\mathrm{P}_{3}$ pulse. In fact, in the raw TR-ISRS signal obtained at $\Delta \mathrm{T}=40$ ps (Figure $2 \mathrm{~B}$, top), we found a 200-fs decay component, which is likely attributable to the population dynamics of this hot ground-state I form. However, this lifetime is too short to provide vibrational bands as sharp as those found in the Fourier spectrum obtained at $\Delta \mathrm{T}=40 \mathrm{ps}$. 


\section{Comparison between TR-ISRS and time-resolved IR spectra of $A^{*}$ and I* forms}

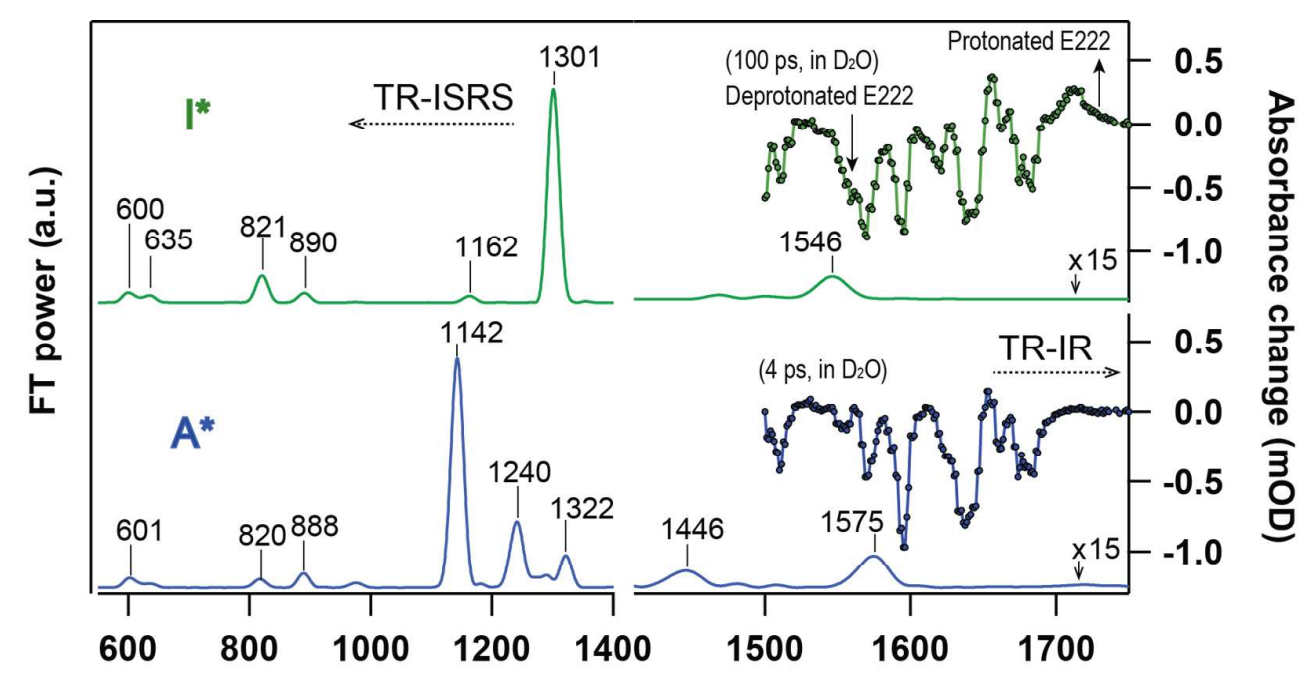

Figure S4. TR-ISRS vibrational spectra of $A^{*}$ (blue, at 0.1 ps in $\mathrm{H}_{2} \mathrm{O}$ ) and $\mathrm{I}^{*}$ (green, at 40 ps in $\mathrm{H}_{2} \mathrm{O}$ ) forms as compared to the reported time-resolved IR (TR-IR) spectra. The TR-IR data of $A^{*}$ and $I^{*}$ forms are adapted from ref. 9.

The time-resolved IR spectra that were reported previously provide complementary information to the vibrational spectra of the $A^{*}$ and I* forms obtained by TR-ISRS. ${ }^{8-11}$ As shown in Figure S4, the time-resolved IR spectra showed the clear change for the protein vibrational bands in the $\sim 1500-1800 \mathrm{~cm}^{-1}$ region upon the $\mathrm{A}^{*} \mathrm{I}^{*}$ conversion, which is associated with the protonation of Glu222 during the ESPT. On the other hand, TR-ISRS details the vibrational modes of the excited-state chromophore (up to $\sim 1550 \mathrm{~cm}^{-1}$ ) transforming from the $\mathrm{A}^{*}$ to the $\mathrm{I}^{*}$ form. 


\section{Temporal change of a transient band of the $I *$ form}

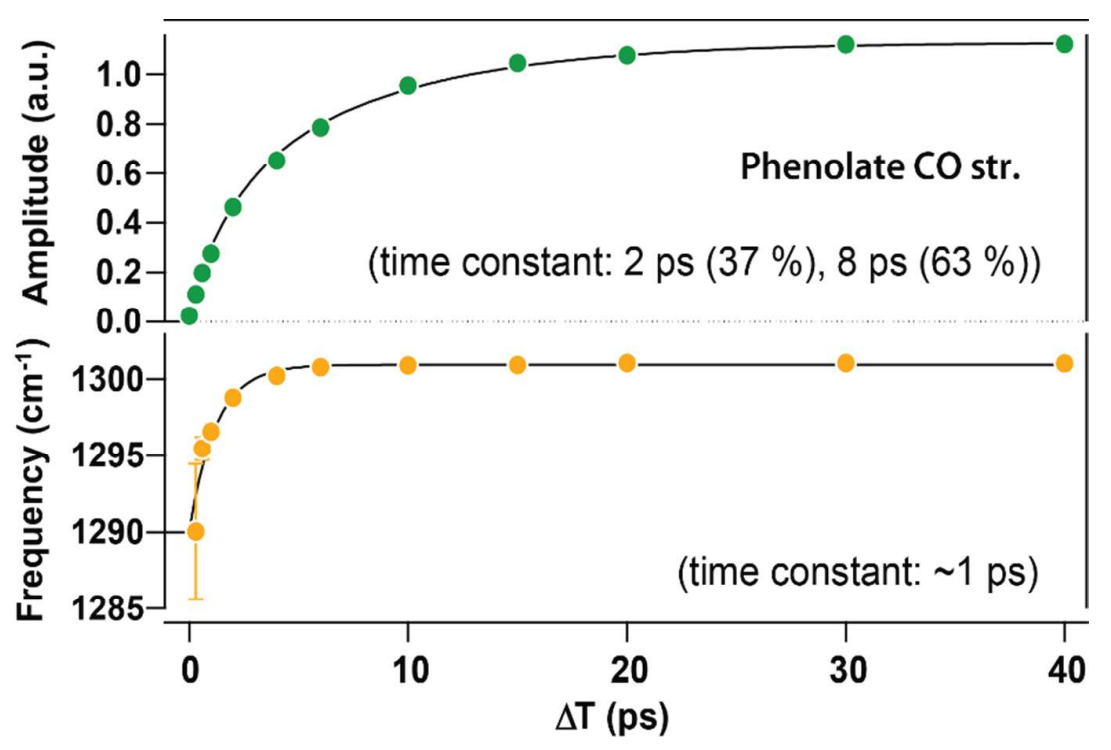

Figure S5. Temporal change in the amplitude (green) and frequency (yellow) of the phenolate CO stretch band of the $\mathrm{I}^{*}$ form. Fit curves (black line) and the obtained time constants are also shown.

The amplitude of phenolate $\mathrm{CO}$ stretch of the I* form shows a temporal rise that is well fit by a bi-exponential function with the time constants of $\sim 2$ ps $(37 \%)$ and $\sim 8$ ps $(63 \%)$. Its vibrational frequency also shows an upshift by $\sim 10 \mathrm{~cm}^{-1}$, which is well fit by a single exponential function with $\sim 1$ ps time constant.

In a previous time-resolved absorption study, ${ }^{12}$ it was proposed that some portion of the $I^{*}$ form is promptly generated by cross-well excitation, whereby the I* form is directly prepared by non-vertical transition through the Franck-Condon factors between the ground-state A form and the excited-state $I^{*}$ form. However, the amplitude of the phenolate $\mathrm{CO}$ stretch band due to the $\mathrm{I}^{*}$ form is zero at the time origin in the TR-ISRS data within the experimental error, indicating that the prompt generation of the I* form is negligible in GFP. This result is consistent with the conclusion of a previous femtosecond time-resolved fluorescence study. ${ }^{13}$ 


\section{Origin of the hot $I *$ form}

In the TR-ISRS experiment, we observed the frequency shift of the CO stretch $\left(\sim 1300 \mathrm{~cm}^{-1}\right)$, which is attributable to the vibrational cooling of the $\mathrm{I}^{*}$ form, and obtained the time constant of $\sim 1 \mathrm{ps}$ (Figure S5). Considering the slower ESPT ( 2 ps $(37 \%), \sim 8$ ps $(63 \%))$ relative to the vibrational cooling, the production of the hot $\mathrm{I}^{*}$ form is not efficient and the concentration is expected to be low. However, the following calculation on kinetics using the experimental parameters shows that the hot $I^{*}$ form is detectable in the early time region.

Using the reaction scheme as below,

$$
\mathrm{A}^{*} \stackrel{k_{1}}{\longrightarrow} \mathrm{I} \mathrm{I}_{\text {hot }} \stackrel{k_{2}}{\longrightarrow} \mathrm{I}^{*} \quad \text { (Scheme S1), }
$$

we obtain the kinetics of $\mathrm{A}^{*}, \mathrm{I}^{*}$ hot, and I* as

$$
\begin{aligned}
& {\left[A^{*}\right]=\left[A^{*}\right]_{0} \cdot \exp \left(-k_{1} t\right),} \\
& {\left[I_{\text {hot }}^{*}\right]=\left[A^{*}\right]_{0} \cdot \frac{k_{1}}{k_{2}-k_{1}}\left\{\exp \left(-k_{1} t\right)-\exp \left(-k_{2} t\right)\right\},} \\
& {\left[I^{*}\right]=\left[A^{*}\right]_{0} \cdot\left\{1+\frac{k_{1} \exp \left(-k_{2} t\right)-k_{2} \exp \left(-k_{1} t\right)}{k_{2}-k_{1}}\right\} .}
\end{aligned}
$$

In this reaction scheme, $k_{1}$ is given as $(4.5 \mathrm{ps})^{-1}$ according to the average time constant of ESPT $(\sim 4.5$ ps) and $k_{2}$ is estimated to be $(1 \mathrm{ps})^{-1}$ from the frequency shift of the CO stretch. Figure S6A shows the calculated temporal profiles of the population of $\mathrm{I}^{*}$ hot, $\mathrm{I}^{*}$, and total $\mathrm{I}^{*}\left(\mathrm{I}^{*}{ }_{\mathrm{hot}}+\mathrm{I}^{*}\right)$. The experimental data are well reproduced by the temporal profile of the total $I^{*}$-form population. Clearly, the contribution of $\mathrm{I}^{*}{ }_{\text {hot }}$ is shown to be significant within 1 ps after photoexcitation (Figure $\mathrm{S} 6 \mathrm{~B}$ ), indicating that the vibrational band due to $\mathrm{I}^{*}$ hot can be observed in this early time region.
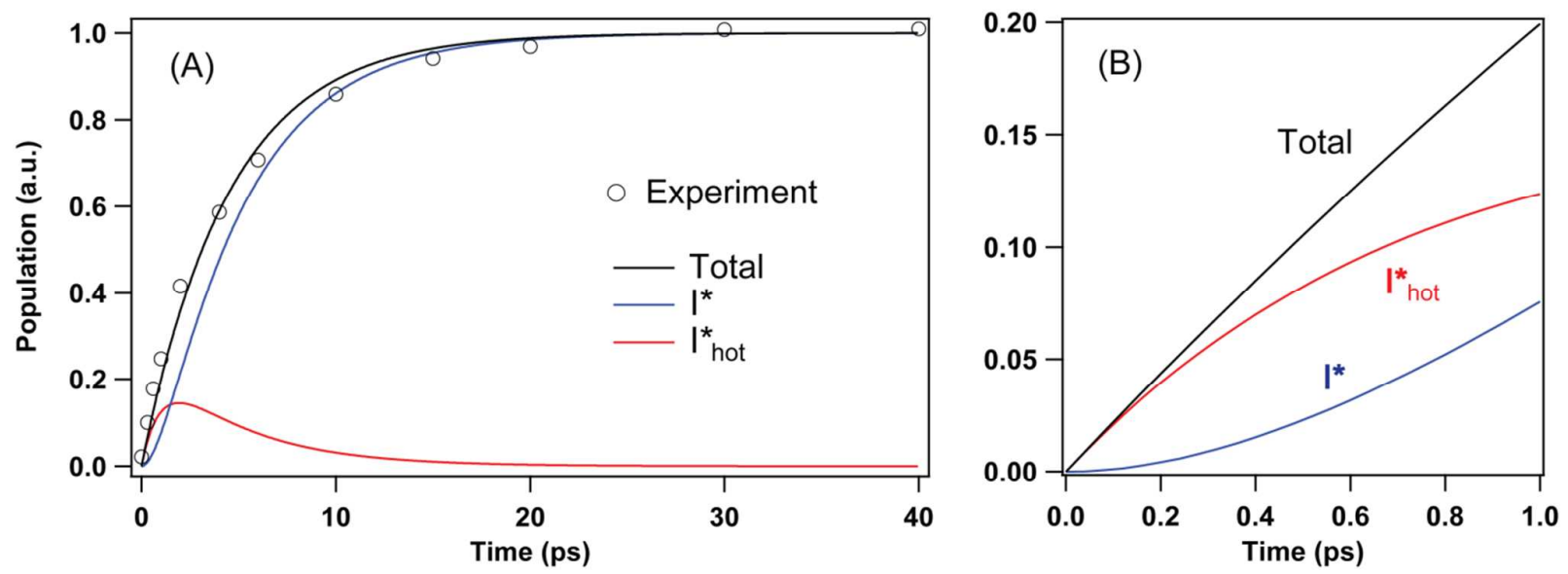

Figure S6. Temporal profiles of $I^{*}$ (blue), $I^{*}{ }_{\text {hot }}$ (red), and the total (black) based on scheme S1. (A) from 0 to $40 \mathrm{ps}$, (B) within $1 \mathrm{ps.}$ 


\section{Comparison of the deviation of the early-time $I *$ formation from the first order kinetics and the low-frequency spectrum of $A^{*}$}
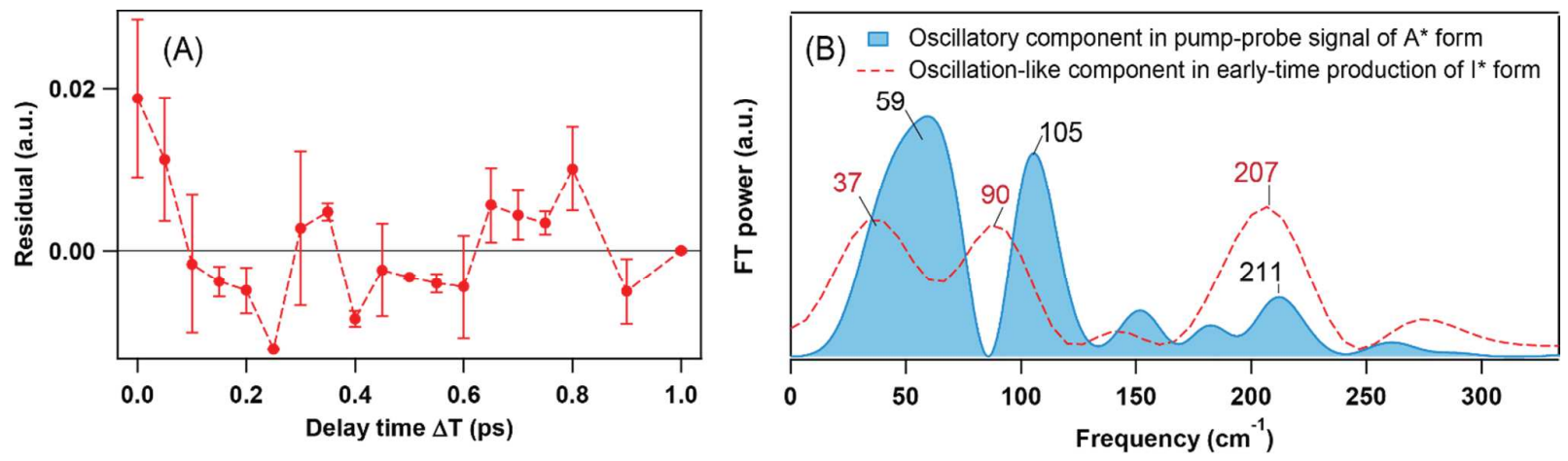

Figure S7. (A) Residual component obtained after fitting the early-time $\mathrm{I}^{*}$ formation shown in Figure 5. (B) Fourier spectra of the oscillatory component of the pump-probe signal of the $A^{*}$ form (blue; cf. Figure 2A, inset) and the modulation component in the early-time formation process of the $\mathrm{I}^{*}$ form (red; cf. Figure 5 , top).

Figure S7A shows the residual component obtained after fitting the early-time I* formation. (This is the same plot as shown in Figure 5 in the main text.) This residual component may exhibit slight modulation against the $\Delta \mathrm{T}$ delay time as its frequency-domain Fourier spectrum (Figure $\mathrm{S} 7 \mathrm{~B}$, red) shows several bands, whose peak frequencies and intensity pattern look similar to those of the low-frequency vibrational spectrum of the $A^{*}$ form (Figure S7B, blue: the same as shown in Figure 2A). This might suggest that a very small portion of the $I^{*}$ formation is driven by the coherent low-frequency motion. However, the modulation amplitude is much smaller than that of the $A^{*}$ signal (phenolic $\mathrm{CH}$ bend), and it is comparable to the experimental uncertainty. In addition, if the residual component really exhibits modulation reflecting the coherent production of the $I^{*}$ form, the modulation should exhibit a damping behavior, as observed for the $\mathrm{A}^{*}$ oscillation. However, such a behavior cannot be recognized, suggesting that the possible coherence contribution is below the noise level of the present experiments even if it exists. In any case, the modulation amplitude of the $I^{*}$ form is at most $1 \%$ of the final amplitude of the $I^{*}$ form generated by the ESPT. Therefore, we can conclude that the low-frequency motion does not play a predominant role in the ESPT reaction of GFP, at least at room temperature. 


\section{Formulation of the amplitude modulation of a high frequency Raman band through the anharmonic coupling with a low-frequency mode}

In the TR-ISRS experiment, the Raman-active vibrations of the $A^{*}$ form are observed under the resonance condition (with both the stimulated emission and excited-state absorption), and their intensities are determined by the relevant Franck-Condon factors of the electronic transition in resonance (under the Condon approximation). ${ }^{14-16}$ Since the Franck-Condon factor is directly related to the displacement $(\Delta)$ between the potential curves of the two electronic states, the modulation of the Raman intensity reflects the modulation of $\Delta$. In the present study, we observed that the amplitude of the phenolic $\mathrm{CH}$ bend oscillates with a frequency of $104 \mathrm{~cm}^{-1}$. This implies that $\Delta$ along the phenolic $\mathrm{CH}$ bend coordinate is modulated with the frequency of $104 \mathrm{~cm}^{-1}$. In the following, we provide a formulation that shows that the anharmonic vibrational coupling with a low-frequency mode gives rise to the displacement modulation for the high-frequency vibrational coordinate, i.e., the phenolic $\mathrm{CH}$ bend.

The two-dimensional anharmonic potential $(V)$, which contains up to the third-order coupling terms between the phenolic $\mathrm{CH}$ bend $Q_{\mathrm{CH}}$ bend (frequency: $\omega_{\mathrm{CH} \text { bend }}$ ) and a low-frequency mode $x$ (frequency: $\omega_{x}$ ), is given by

$V=\frac{1}{2} \omega_{\mathrm{CH} \text { bend }} Q_{\mathrm{CHbend}}^{2}+\alpha Q_{\mathrm{CHbend}}^{2} x+\beta Q_{\mathrm{CHbend}} x^{2}+\frac{1}{2} \omega_{x} x^{2}$.

After completing the square for the $Q_{\text {CHbenc }}$ coordinate, the anharmonic potential $V$ is expressed as

$$
\begin{aligned}
V & =\frac{1}{2}\left(\omega_{\mathrm{CH} \text { bend }}+2 \alpha x\right) \cdot\left(Q_{\mathrm{CH} \text { bend }}+\frac{\beta x^{2}}{\omega_{\mathrm{CH} \text { bend }}+2 \alpha x}\right)^{2}+\frac{1}{2} \omega_{x} x^{2}-\frac{\beta^{2} x^{4}}{2\left(\omega_{\mathrm{CH} \text { bend }}+2 \alpha x\right)} \\
& \approx \frac{1}{2}\left(\omega_{\mathrm{CH} \text { bend }}+2 \alpha x\right) \cdot\left(Q_{\mathrm{CH} \text { bend }}+\frac{\beta x^{2}}{\omega_{\mathrm{CH} \text { bend }}}\right)^{2}+\frac{1}{2} \omega_{x} x^{2}-\frac{\beta^{2} x^{4}}{2 \omega_{\mathrm{CH} \text { bend }}}
\end{aligned}
$$

where an approximation $\alpha x<<\omega_{\text {CHbend }}$ is used in the second line. The equation (2) shows that the third-order coupling terms perturb the harmonic potential of $Q_{\mathrm{CHbend}}$ in two ways: the coupling term $\alpha Q_{\mathrm{CH} \text { bend }}^{2} x$ changes the frequency $\left(\omega_{\mathrm{CHbend}}\right)$ by $20 x$, and the other term $\beta Q_{\mathrm{CHbend}} x^{2}$ shifts the potential minimum by $-\frac{\beta x^{2}}{\omega_{\mathrm{CH} \text { bend }}}$ along the $Q_{\mathrm{CH} \text { bend }}$ coordinate.

In the presence of the anharmonic coupling, the potential displacement of the $Q_{\mathrm{CH} \text { bend }}\left(\Delta_{\mathrm{CH} \text { bend }}\right)$ 
between two electronic states (e.g., $\mathrm{S}_{0}$ and $\mathrm{S}_{1}$ states), which are relevant to the resonance Raman process, can be calculated as illustrated in Figure S8. As shown in the figure, the anharmonic vibrational potentials of the two electronic states are relatively shifted by $\Delta_{Q}$ and $\Delta_{\text {x }}$ along the $Q_{\mathrm{CH} \text { bend }}$ and $x$ coordinates, respectively, because both $Q_{\mathrm{CHbend}}$ and $x$ are Franck-Condon active. Under harmonic approximation, the potential displacement along the phenolic $\mathrm{CH}$ bend $\left(\Delta_{\mathrm{CH} \text { bend }}\right)$ is simply $\Delta_{Q}$. However, due to the anharmonic coupling with a low-frequency mode $x$, the additional shift of $-\frac{\beta x^{2}}{\omega_{\mathrm{CH} \text { bend }}}$ is induced in the vibrational potential of $Q_{\mathrm{CHbend}}$ on each electronic state. For this reason, $\Delta_{\text {CHbend }}$ between the two electronic states is written as

$\Delta_{\mathrm{CH} \text { bend }} \approx \Delta_{Q}+\frac{\beta x_{\mathrm{S} 0}^{2}}{\omega_{\mathrm{CH} \text { bend }}}-\frac{\beta x_{\mathrm{S} 1}^{2}}{\omega_{\mathrm{CH} \text { bend }}}$,

$x_{\mathrm{S} 0}=x_{\mathrm{S} 1}+\Delta_{x}$

where $x_{\mathrm{S} 0}$ and $x_{\mathrm{S} 1}$ represent the positions of $x$ in the $\mathrm{S}_{0}$ and $\mathrm{S}_{1}$ states, respectively, and the same $\omega_{\mathrm{CHbend}}$ and $\beta$ are used for the two electronic states, for simplicity.

Then, by substituting eq.4 into eq.3, we obtain

$$
\Delta_{\mathrm{CH} \text { bend }} \approx \Delta_{Q}+\frac{\beta}{\omega_{\mathrm{CH} \text { bend }}}\left(\Delta_{x}^{2}+2 \Delta_{x} x_{\mathrm{S} 1}\right) .
$$

$\Delta_{\text {CHbend }}$ contains the additional term that depends on the position of $x$ and the potential displacement $\Delta_{x}$.

In the TR-ISRS experiment, the low-frequency mode $x$ is coherently excited in the $\mathrm{S}_{1}$ state by $\mathrm{P}_{1}$ pulse, which can be treated as the classical vibrational motion with frequency $\omega_{x}$

$$
x_{\mathrm{S} 1}=A \cos \left(\omega_{x} t+\phi\right) \text {, }
$$

where $A$ and $\phi$ are the amplitude and initial phase of the low-frequency vibration, respectively.

With substituting eq. 6 into eq. 5 , we obtain,

$$
\Delta_{\mathrm{CH} \text { bend }} \approx \Delta_{Q}+\frac{\beta}{\omega_{\mathrm{CH} \text { bend }}}\left\{\Delta_{x}^{2}+2 A \Delta_{x} \cos \left(\omega_{x} t+\phi\right)\right\} \text {. }
$$

The equation (7) demonstrates that $\Delta_{\text {CHbend }}$ is modulated with the low frequency $\omega_{x}$ through the 
anharmonic coupling. This demonstrates that the Raman intensity of the $Q_{\mathrm{CH}}$ bend mode exhibits the low-frequency modulation with the frequency $\omega_{x}$ through the anharmonic coupling. As shown in Figure $4 \mathrm{~A}$, we observed $\sim \pm 30 \%$ modulation for the FT amplitude of the phenolic $\mathrm{CH}$ bend. On the basis of eq.7, this large amplitude oscillation indicates the large coupling constant $\beta$ for the specific pair of the phenolic $\mathrm{CH}$ bend and the $104-\mathrm{cm}^{-1}$ modes.

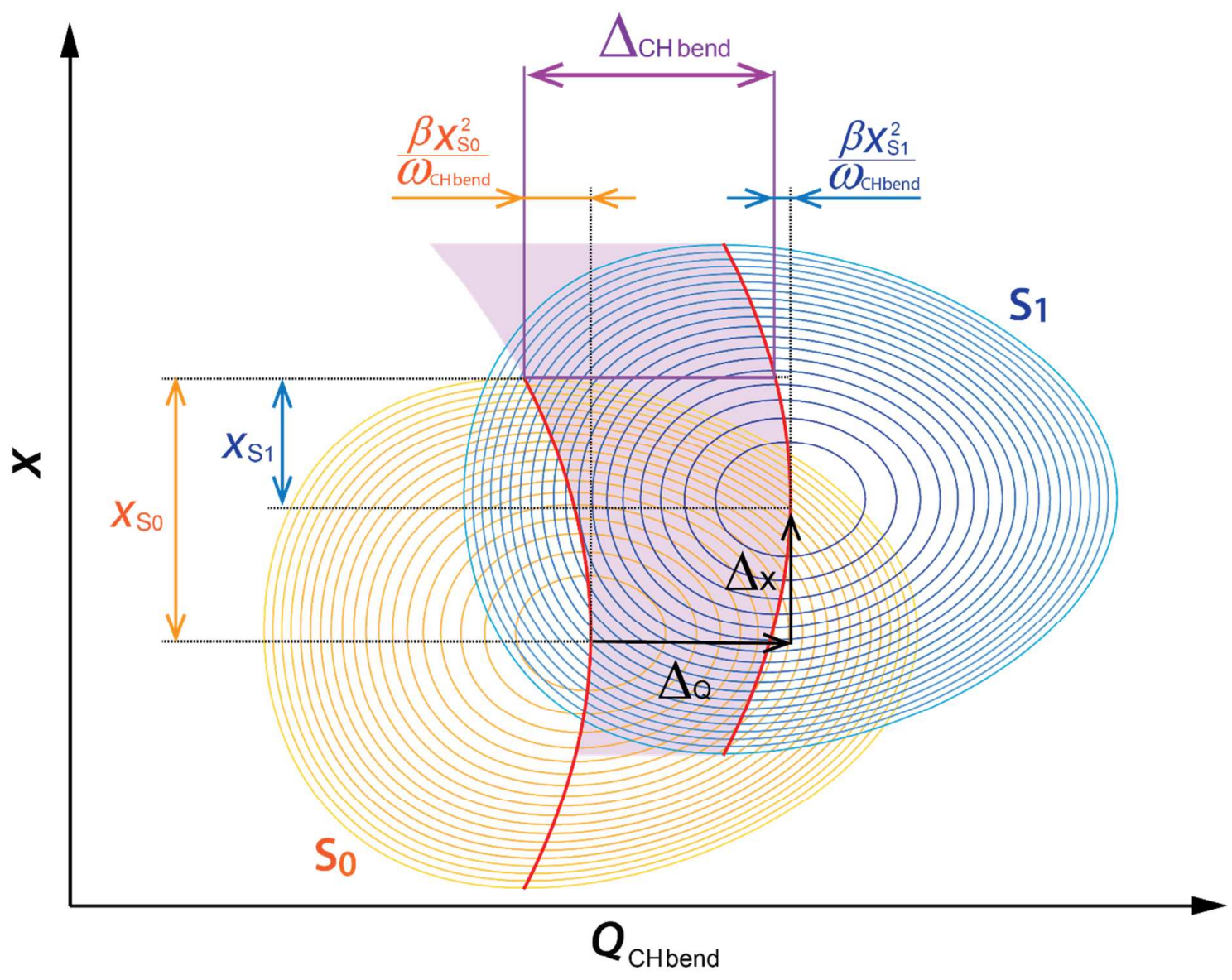

Figure S8. Illustration of two-dimensional vibrational potentials of the $S_{0}$ (yellow) and $S_{1}$ states (blue) which consist of the phenolic $\mathrm{CH}$ bend $\left(\mathrm{Q}_{\mathrm{CH} \text { bend }}\right)$ and the low-frequency mode $(\mathrm{x})$. The global minima of the two potentials are displaced by $\Delta_{\mathrm{Q}}$ along the phenolic $\mathrm{CH}$ bend coordinate $\mathrm{Q}_{\mathrm{CH} \text { bend }}$ and by $\Delta_{\mathrm{x}}$ along the low-frequency mode $x$. Red curves depict the changes in the vibrational potential minima of $Q_{C H}$ bend, which are expressed as $-\beta \mathrm{x}_{\mathrm{SO}_{0}}{ }^{2} / \omega_{\mathrm{CH}}$ bend and $-\beta \mathrm{x}_{\mathrm{S} 1}{ }^{2} / \omega_{\mathrm{CH}}$ bend for the $\mathrm{S}_{0}$ and $\mathrm{S}_{1}$ states, respectively. The purple area between the two red curves represents the potential displacement along $Q_{\mathrm{CH}}$ bend coordinate $\left(\Delta_{\mathrm{CH}}\right.$ bend $)$ between the two electronic states. 


\section{Supplementary references}

(1) Heim, R.; Tsien, R. Y. Curr. Biol. 1996, 6, 178.

(2) Hosoi, H.; Hazama, S.; Takeda, Y. Chem. Phys. Lett. 2015, 618, 186.

(3) Takeuchi, S.; Ruhman, S.; Tsuneda, T.; Chiba, M.; Taketsugu, T.; Tahara, T. Science 2008, $322,1073$.

(4) Kuramochi, H.; Takeuchi, S.; Yonezawa, K.; Kamikubo, H.; Kataoka, M.; Tahara, T.; submitted

(5) Kuramochi, H.; Takeuchi, S.; Tahara, T.; submitted

(6) Fang, C.; Frontiera, R. R.; Tran, R.; Mathies, R. A. Nature 2009, 462, 200.

(7) Kennis, J. T.; Larsen, D. S.; van Stokkum, I. H.; Vengris, M.; van Thor, J. J.; van Grondelle, R. Proc. Natl. Acad. Sci. USA 2004, 101, 17988.

(8) van Thor, J. J.; Zanetti, G.; Ronayne, K. L.; Towrie, M. J. Phys. Chem. B 2005, 109, 16099.

(9) Stoner-Ma, D.; Melief, E. H.; Nappa, J.; Ronayne, K. L.; Tonge, P. J.; Meech, S. R. J. Phys. Chem. B 2006, 110, 22009.

(10) Stoner-Ma, D.; Jaye, A. A.; Matousek, P.; Towrie, M.; Meech, S. R.; Tonge, P. J. J. Am. Chem. Soc. 2005, 127, 2864.

(11) Di Donato, M.; van Wilderen, L. J.; Van Stokkum, I. H.; Stuart, T. C.; Kennis, J. T.; Hellingwerf, K. J.; van Grondelle, R.; Groot, M. L. Phys Chem Chem Phys 2011, 13, 16295.

(12) Winkler, K.; Lindner, J.; Subramaniam, V.; Jovin, T. M.; Vöhringer, P. Phys. Chem. Chem. Phys. 2002, 4, 1072.

(13) Kondo, M.; Heisler, I. A.; Stoner-Ma, D.; Tonge, P. J.; Meech, S. R. J. Am. Chem. Soc. 2010, $132,1452$.

(14) Albrecht, A. C. J. Chem. Phys. 1961, 34, 1476.

(15) Heller, E. J.; Sundberg, R.; Tannor, D. J. Phys. Chem. 1982, 86, 1822.

(16) Clark, R. J. H.; Dines, T. J. Angew. Chem. 1986, 98, 131. 